\title{
Expression analysis of the osteoarthritis genetic susceptibility mapping to the matrix Gla protein gene MGP
}

\author{
Colin Shepherd ${ }^{*}$ (D, Abigail E. Reese, Louise N. Reynard and John Loughlin
}

\begin{abstract}
Background: Osteoarthritis $(\mathrm{OA})$ is a common disease of older individuals that impacts detrimentally on the quality and the length of life. It is characterised by the painful loss of articular cartilage and is polygenic and multifactorial. Genome-wide association scans have highlighted over 90 osteoarthritis genetic signals, some of which reside within or close to highly plausible candidate genes. An example is an association to polymorphisms within and adjacent to the matrix Gla protein gene MGP. We set out to undertake a functional study of this gene.

Methods: Nucleic acid was extracted from cartilage, infrapatellar fat pad, synovium, trabecular bone, trapezium and peripheral whole blood from OA patients and also from mesenchymal stem cells (MSCs) subjected to chondrogenesis. Expression of MGP was measured by quantitative PCR (qPCR), RNA-sequencing and allelic expression imbalance (AEI) analysis. Matrix Gla protein was depleted in chondrocytes by knocking down MGP expression using RNA interference (RNAi) and the effect on a range of genes assessed by qPCR.

Results: MGP is expressed in joint tissues, blood and chondrocytes cultured from MSCs. There is a higher expression in diseased versus non-diseased cartilage. Polymorphisms that are associated with OA also correlate with the expression of MGP, with the OA risk-conferring allele showing significantly reduced expression in cartilage, fat pad and synovium but increased expression in blood. Depletion of Matrix Gla protein had a significant effect on the majority of genes tested, with an increased expression of catabolic genes that encode enzymes that degrade cartilage.

Conclusions: MGP expression is subject to cis-acting regulators that correlate with the $\mathrm{OA}$ association signal. These are active in a range of joint tissues but have effects which are particularly strong in cartilage. An opposite effect is observed in blood, highlighting the context-specific nature of the regulation of this gene's expression. Recapitulation of the genetic deficit in cartilage chondrocytes is pro-catabolic.
\end{abstract}

Keywords: Osteoarthritis, Genetic association signal, MGP, Allelic expression, RNA interference, Knockdown

\section{Introduction}

Osteoarthritis (OA) is a highly polygenic musculoskeletal disorder that is common in most populations and principally affects older individuals. It is characterised by a gradual, focal loss of the articular cartilage with concurrent and consequential alterations to other joint tissues [1]. Patients initially present with chronic joint pain and loss of normal joint function with surgical intervention often required in severe cases of the disease. OA therefore impacts on the quality of life, but it can also shorten the length of life [2].

\footnotetext{
* Correspondence: cshep1987@gmail.com

Skeletal Research Group, Institute of Genetic Medicine, Newcastle University, International Centre for Life, Newcastle upon Tyne NE1 3BZ, UK
}

Genome-wide association scans (GWAS) have reported over 90 independent genome-wide significant risk alleles for OA [3-7]. Most of these studies have focused on hand, knee or hip OA, with disease at these sites having a particularly large impact on the health of the patient and on health care systems.

The principal translational goal of OA GWAS studies is the generation of new knowledge regarding disease aetiology that can then be directed to treatment development. In this regard, the association with hand $\mathrm{OA}$ of functional polymorphism at the MGP locus was very noteworthy [8]. This gene codes for matrix Gla protein, which is secreted by cartilage chondrocytes into the synovial joint space,

(C) The Author(s). 2019 Open Access This article is distributed under the terms of the Creative Commons Attribution 4.0 International License (http://creativecommons.org/licenses/by/4.0/), which permits unrestricted use, distribution, and 
where it regulates the levels of free calcium via the affinity of its $\gamma$-carboxyglutamic acid (Gla) residues for calcium ions [9]. In so doing, matrix Gla protein inhibits ectopic calcification of the joint. The OA association was to single nucleotide polymorphism (SNP) rs4764133 $(\mathrm{C}>\mathrm{T})$, which is located upstream of MGP and which correlated with altered expression of the gene in cartilage; the OA-risk conferring $\mathrm{T}$ allele of rs4764133 showed reduced expression relative to the non-risk $C$ allele [8]. This implies that the genetic susceptibility at this locus acts by lowering the level of MGP expression, which leads to reduced amounts of matrix Gla protein. This would be permissive to ectopic cartilage calcification and as such may be the mechanism by which this association signal acts to increase OA risk. This genetic data supports the application in OA of compounds that could attenuate this calcification, such as vitamin $\mathrm{K}$ which is involved in the biosynthesis of Gla-rich proteins [10].

The compelling and potentially translatable nature of the MGP genetic association report prompted us to undertake a more detailed molecular analysis of this signal. We set out to replicate and then expand on the MGP expression study into other joint tissues and cells from patients. We also modelled the impact of the rs4764133 risk-conferring allele by knocking down $M G P$ in cartilage chondrocytes and assessing effects on anabolic, catabolic, and hypertrophic genes.

\section{Materials and methods \\ OA patients}

Joint tissue samples were obtained from patients undergoing orthopaedic surgical procedures at the Newcastle upon Tyne NHS Foundation Trust hospitals. Samples were collected from two categories of patient: (1) those with primary hip or knee OA who had undergone joint replacement surgery and (2) those with primary hand OA who had undergone a trapeziectomy. In total, we accessed and studied tissue samples from a total of OA 165 patients.

For knee and hip OA patients, we collected macroscopically normal cartilage (distal from the lesion and therefore avoiding areas of fibrillated tissue), infrapatellar fat pad, synovium and trabecular bone (which were taken from non-sclerotic areas). For some of our knee and hip patients, more than one joint tissue type was available for analysis. For hand OA patients, cartilage could not be separated from subchondral bone due to the small size of the trapeziectomy samples, and as such, subchondral bone with its attached cartilage (osteochondral samples) was collected. We also collected whole peripheral blood samples from some of our hip and knee OA patients just prior to their surgery, using EDTA vacutainers for DNA extraction and Tempus $^{\text {Tix }}$ tubes for RNA extraction (ThermoFisher Scientific). Further details regarding the patients can be found in Additional file 1: Table S1.

\section{Nucleic acid extractions}

Tissue samples were stored at $-80{ }^{\circ} \mathrm{C}$ and ground to a powder using a mixermill (Retsch Limited) under liquid nitrogen. For cartilage, bone and trapeziectomy samples, RNA was extracted using TRIzol (Life Technologies) and DNA was extracted using the E.Z.N.A. Tissue DNA isolation kit (Omega Biotek, VWR). For synovium and fat pad, DNA and RNA were extracted using the E.Z.N.A. DNA/ RNA isolation kit (Omega Biotek, VWR). For blood, DNA was extracted using the QIAamp DNA blood mini kit (Qiagen) and RNA extracted using the Tempus ${ }^{\mathrm{Tm}}$ Spin RNA isolation kit (ThermoFisher Scientific).

\section{Quantitative gene expression}

cDNA synthesis and quantitative PCR (qPCR) were performed as described previously [11]. Predesigned TaqMan assays (Integrated DNA Technologies) or assays designed using the Roche probe library system were used to quantify expression of the housekeeping genes HPRT1, 18S and GAPDH and 12 target genes. The relative gene expression was calculated by the $2^{-\Delta \mathrm{Ct}}$ method, where $\Delta \mathrm{Ct}$ is the mean $\mathrm{Ct}$ value of the three housekeeping genes subtracted from the $\mathrm{Ct}$ value of the target gene of interest. For target gene $R U N X 2$, two qPCR assays were used: one targeting exons $6-7$, measuring both the main isoforms of the gene (termed RUNX2all), and one targeting exons $1-2$, which measures the longer isoform of the gene (termed RUNX2long) [12].

\section{Mesenchymal stem cell (MSC) chondrogenesis}

Immortalised human MSCs derived from adipose tissue (SCRC-4000, ATCC) were subjected to V-bottom 96-wellplate pellet chondrogenesis for 21 days, as described previously [13]. Human bone marrow MSCs from a female donor aged 24 years (Lonza Biosciences) were cultured, phenotype tested and subjected to V-bottom 96-well-plate pellet chondrogenesis for 7 days, as described previously [14]. In each case, three pellets were analysed at each time point, representing three independent differentiations. RNA was extracted from each pellet using TRIzol (Life Technologies), cDNA synthesised and MGP expression measured by qPCR with three technical repeats per cDNA.

\section{RNA-sequencing (RNA-seq)}

The expression of MGP was assessed using RNA-seq data that we had previously generated from the cartilage of ten hip OA patients and six patients who had undergone hip replacement due to a neck-of-femur (NOF) intracapsular fracture [15]. The NOF patients showed no signs or symptoms of hip OA, and their cartilage was macroscopically intact and with no lesions. Transcripts per million (TPM) values were extracted using $R$ (http:// www.R-project.org/) and visualised using the ggplot2 library in R. Differential expression analysis between OA 
and NOF was carried out with the Bioconductor package DESeq2 [16].

\section{Genotyping}

We chose to analyse MGP transcript SNP rs4236 (T > $\mathrm{C}$, OA risk allele $=\mathrm{C}$, minor allele frequency $=0.37$ ) as the difference in expression between its risk and nonrisk alleles was the largest of those SNPs investigated in the original study [8]. The SNP was genotyped by pyrosequencing using the primers listed in Additional file 2: Table S2. The assay was designed using PyroMark assay design 2.0 (Qiagen), and the sequencing was performed using the PyroMark Q24 Advanced platform (Qiagen).

\section{Allelic expression imbalance (AEI)}

AEI at rs4236 heterozygotes was quantified by pyrosequencing, using the methodology described above and the same primers. The sequences were generated automatically and an output of allelic ratio was produced using PyroMark Advanced software (Qiagen). For each cDNA and DNA sample from a heterozygote, PCR reactions were formed in triplicate. Samples were excluded from the analysis if the values between the PCR replicates differed by $>5 \%$. The respective cDNA and DNA were analysed concurrently and allelic expression of cDNA was normalised to its corresponding DNA.

\section{RNA interference (RNAi)}

Primary human articular chondrocytes (HACs) were isolated by enzymatic digestion of OA knee cartilage, cultured, and RNAi was then performed, essentially as described previously $[11,17]$. The cells were seeded in 6-well plates at a density of 350,000 cells per well. After $24 \mathrm{~h}$, cells were transfected with $50 \mathrm{nM}$ Dharmacon ON-TARGETplus SMARTpool small interfering RNA (siRNA) targeted against MGP (L-009770-00) or a nontargeting scrambled siRNA control (D-001810-10-20). Cells were harvested $48 \mathrm{~h}$ after transfection, and RNA and protein were extracted using the Nucleospin RNA/ protein kit (Macherey-Nagel, supplied by ThermoFisher). Gene expression was assessed by qPCR using cDNA synthesised from RNA extracted from each well, with a minimum of four technical repeats per gene analysed. For immunoblot analysis of matrix Gla protein depletion, $10 \mu \mathrm{g}$ of total protein was resolved on $10 \%$ (w/v) SDS-polyacrylamide gels. Blots were probed with anti-matrix Gla protein ( $\alpha$-MGP; Proteintech 10734-1AP) or anti-GAPDH $(\alpha-$ GAPDH; Cell Signalling Technologies) antibodies. Matrix Gla protein depletion was quantified using ImageJ software [18] by normalising to levels of $\alpha$-GAPDH.

\section{Statistical analyses}

For quantitative gene expression analyses, $P$ values were calculated using a Mann-Whitney 2-tailed exact test. For RNA-seq, hypothesis testing was performed using the DESeq2 implementation of the Wald test. For AEI, $P$ values were calculated using a Mann-Whitney 2-tailed exact test. For gene expression analyses in the RNAi experiment, $P$ values were calculated using a Student's 2-tailed $t$ test. For quantification of MGP protein abundance, $P$ values were calculated using a Student's 2-tailed $t$ test.

\section{Results \\ Expression of MGP}

Using qPCR, we initially assessed the expression of $M G P$ in five joint tissues from our OA patients: cartilage $(n=$ $22)$, fat pad $(n=9)$, synovium $(n=13)$, trabecular bone $(n=14)$, and trapezium $(n=8)$. We also examined expression in peripheral blood ( $n=15$ patients). MGP was expressed in all samples, with the highest level being in cartilage and the lowest in blood (Fig. 1a). We next compared the level of MGP expression between hip cartilage from OA $(n=10)$ and NOF $(n=6)$ patients in our RNAseq data. The gene was expressed in both patient groups with a non-significant $(P=0.08)$ higher level in OA (Fig. 1b). Using this RNA-seq data, we then assessed the relative expression levels of the transcript isoforms of MGP. The gene has four isoforms, three of which are protein coding (isoforms 201, 203 and 204) and one which is not (isoform 202). All were expressed in cartilage, with isoform 203, which codes for the canonical form of the protein, being most abundant (Fig. 1c). We next assessed the expression of MGP during pellet chondrogenesis, which was performed on an immortalised human MSC cell line (Fig. 1d) and on primary MSCs from a human donor (Fig. 1f). The gene showed an induction in both cell types, with a particularly striking increase in expression during chondrogenesis in the cell line.

\section{Allelic expression imbalance}

In the GWAS report for $M G P$, the authors used allelic expression imbalance (AEI) analysis to demonstrate that the OA risk conferring $\mathrm{T}$ allele of rs4764133 correlated with reduced expression of the gene in cartilage [8]. To do this, they studied three MGP transcript SNPs (rs4236, rs1049897 and rs1800801) that are in high linkage disequilibrium (LD) with each other and with rs4764133 (all pair wise $r^{2}$ values $>0.85$ ). Each transcript SNP had shown significant evidence of AEI. We chose to use rs4236 ( $\mathrm{T}>\mathrm{C} ; r^{2}=0.89$ relative to rs4764133; $\mathrm{T}$ allele of rs4764133 is equivalent to the $\mathrm{C}$ allele of rs4236), the SNP that showed the largest degree of AEI in that initial report (39.6\% of the OA risk-conferring C allele versus $60.4 \%$ of the non-risk $\mathrm{T}$ allele). This SNP is located within the final exon of $M G P$ and is present in 

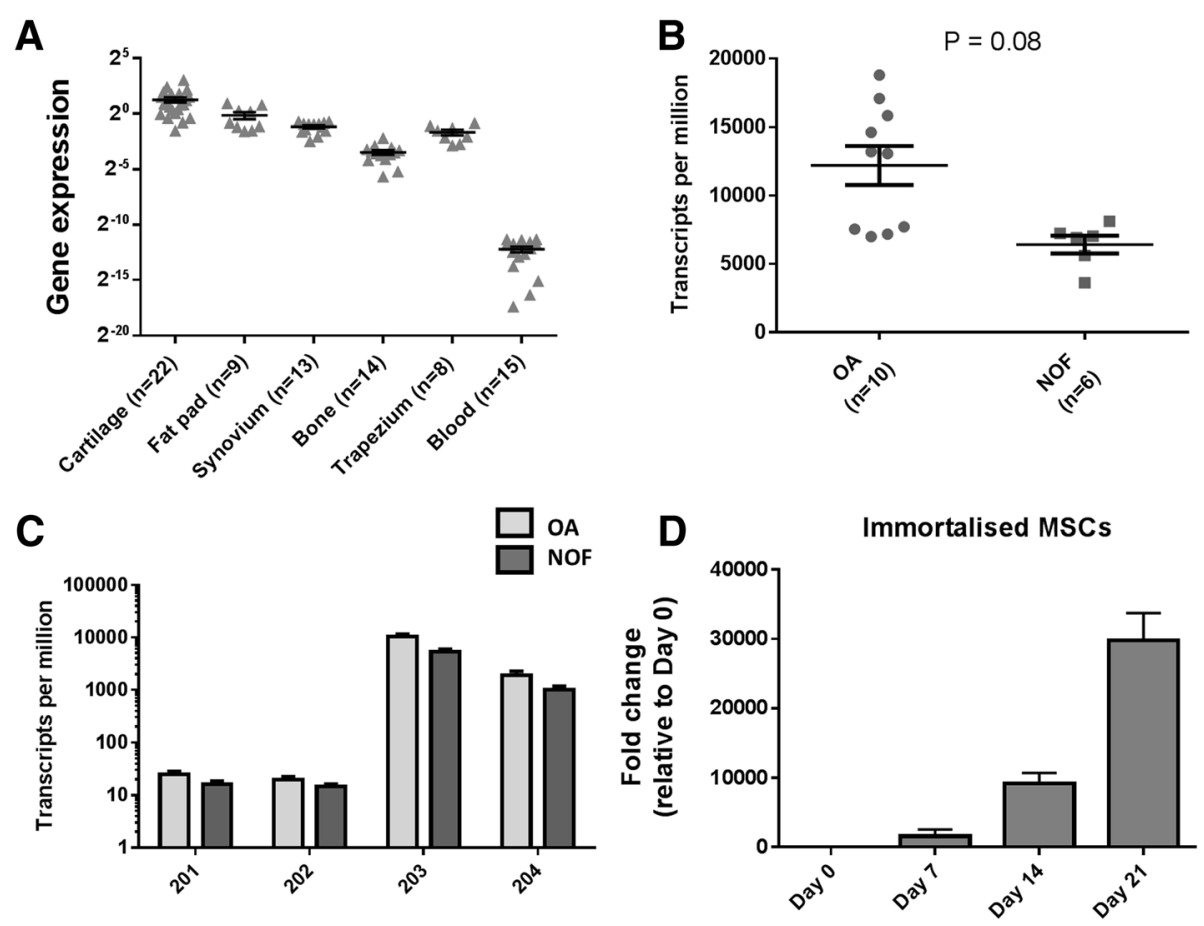

Ensembl transcript isoform ID

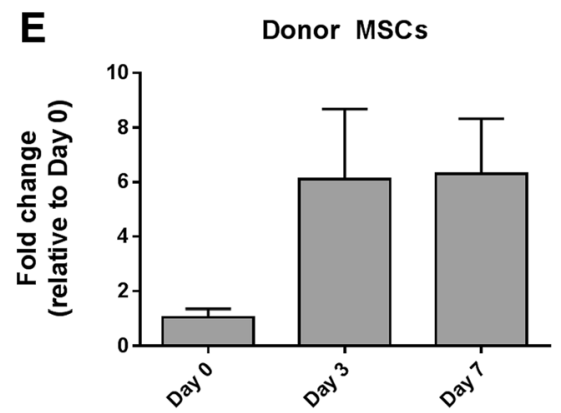

Fig. 1 Expression analysis of MGP in multiple tissue samples from patients and during chondrogenesis. a Expression of MGP was measured by qPCR in OA patient cartilage, infrapatellar fat pad, synovium, trabecular bone, trapezium and blood. b Expression of MGP was measured in hip cartilage from OA patients and patients with a neck-of-femur (NOF) fracture as controls, using RNA-sequencing data. c Expression of MGP transcript isoforms (designated by their Ensembl database identification numbers) was measured using the same RNA-sequencing data. $\mathbf{d}$ Expression of MGP during chondrogenesis of immortalised human MSCs. e Expression of MGP during chondrogenesis of bone marrow MSCS from a human donor. In all panels, horizontal lines with bars show the mean \pm standard error of the mean (SEM)

all four transcript isoforms. We genotyped our patients for rs4236 to identify heterozygotes. We then investigated AEI in cartilage ( $n=30$ patients), fat pad ( $n=26$ patients), synovium ( $n=28$ patients), trapezium ( $n=6$ patients) and blood ( $n=19$ patients) (Fig. 2). In cartilage, we observed AEI that was comparable to that seen in the original report; a mean AEI ratio $(\mathrm{C} / \mathrm{T})$ of 0.69 , equivalent to an average of $40.8 \%$ of the $C$ allele in the 30 patients combined $\left(P=2 \times 10^{-12}\right)$. In fat pad and synovium, we also observed a significant AEI, although not as striking as that seen in cartilage: a mean AEI ratio of 0.81 , equivalent to $44.8 \%$ of the $C$ allele, for fat pad
$\left(P=1 \times 10^{-5}\right)$, and a mean AEI ratio of 0.84 , equivalent to $45.7 \%$ of the $C$ allele, for synovium $\left(P=8 \times 10^{-4}\right)$. In trapezium, there was a non-significant $(P=0.18)$ mean AEI ratio of 0.89 , equivalent to $47.1 \%$ of the $\mathrm{C}$ allele, whilst in blood, the mean AEI ratio was in the opposite direction to that seen in the joint tissues: 1.19 , which is equivalent to $54.3 \%$ of the $C$ allele $(P=0.006)$.

To highlight this difference in MGP AEI between tissues, we plotted together the mean AEI values for the five tissue types tested (Fig. 3a). This clearly shows the variation in AEI between the tissues, with the risk allele showing reduced expression in cartilage toward increased expression 


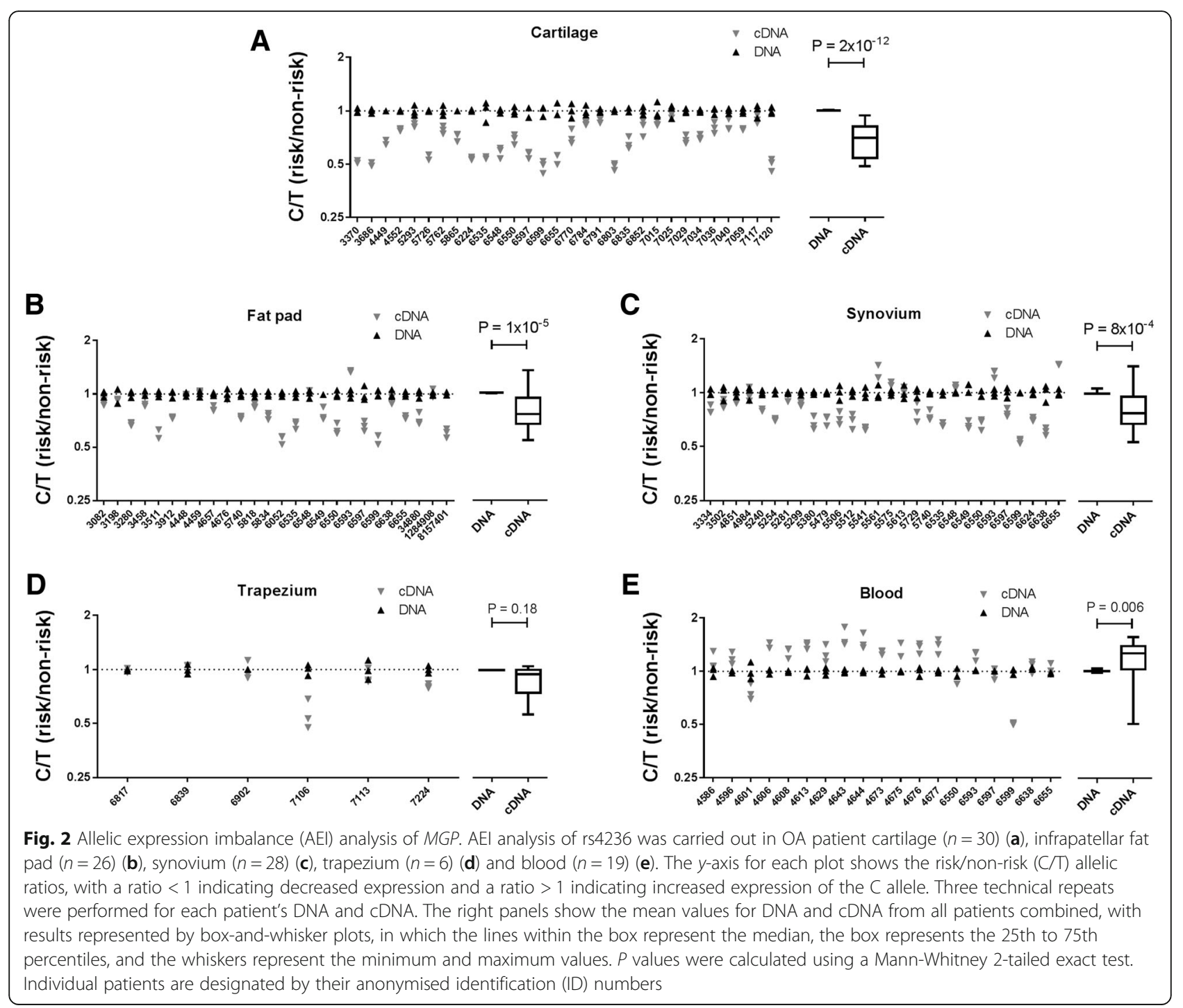

in blood. To emphasise the variability of AEI in tissues from the same patient, we next plotted the data for those patients in whom we had studied two or more tissues (Fig. 3b). There were 11 such patients, and there is clear variability in the $\mathrm{C} / \mathrm{T}$ ratio between the different tissues tested for most of these, with patient 6597 demonstrating particularly clearly the inter-tissue variability of the ratio.

\section{Knockdown of MGP and matrix Gla protein}

Having confirmed that the OA risk-conferring $\mathrm{T}$ allele of rs4764133/C allele of rs4236 correlates with decreased expression of MGP in joint tissues, we next modelled this effect. Chondrocytes were isolated from the knee cartilage of nine OA patients and then cultured in monolayer and subjected to MGP knockdown by RNAi. Compared to the effects of the scrambled siRNA control, siRNA targeting $M G P$ achieved a mean knockdown at the MGP mRNA level of $93 \%(P<0.0001)$ (Fig. 4 a) with a $43 \%$ reduction at the protein level $(P<0.01)$ (Fig. $4 b, c)$.

We then assessed the effect of this knockdown on the expression of a panel of genes that encompass cartilage homeostasis: three chondroprotective/anabolic genes (SOX9, COL2A1 and ACAN), five genes involved in cartilage hypertrophy (RUNX2, ALPL, COL1A1, COL10A1 and $V E G F A)$, three catabolic genes (MMP13, ADAMTS4 and $A D A M T S 5)$ and the anti-apoptotic gene $B C L 2$. Depletion of $M G P$ correlated with a significant $(P \leq 0.05)$ alteration in the expression of nine of the 12 genes tested (Fig. 4d). There was a reduction in expression of the longer isoform of RUNX2 (encodes runt-related transcription factor 2 ; fold change $=0.67, P<0.05), A L P L$ (encodes alkaline phosphatase; fold change $=0.44, P<$ 0.01 ) and COL1A1 (encodes the $\alpha 1$ polypeptide chain of type I collagen; fold change $=0.38, P<0.01$ ), and a significant increase in the expression of SOX9 (encodes 


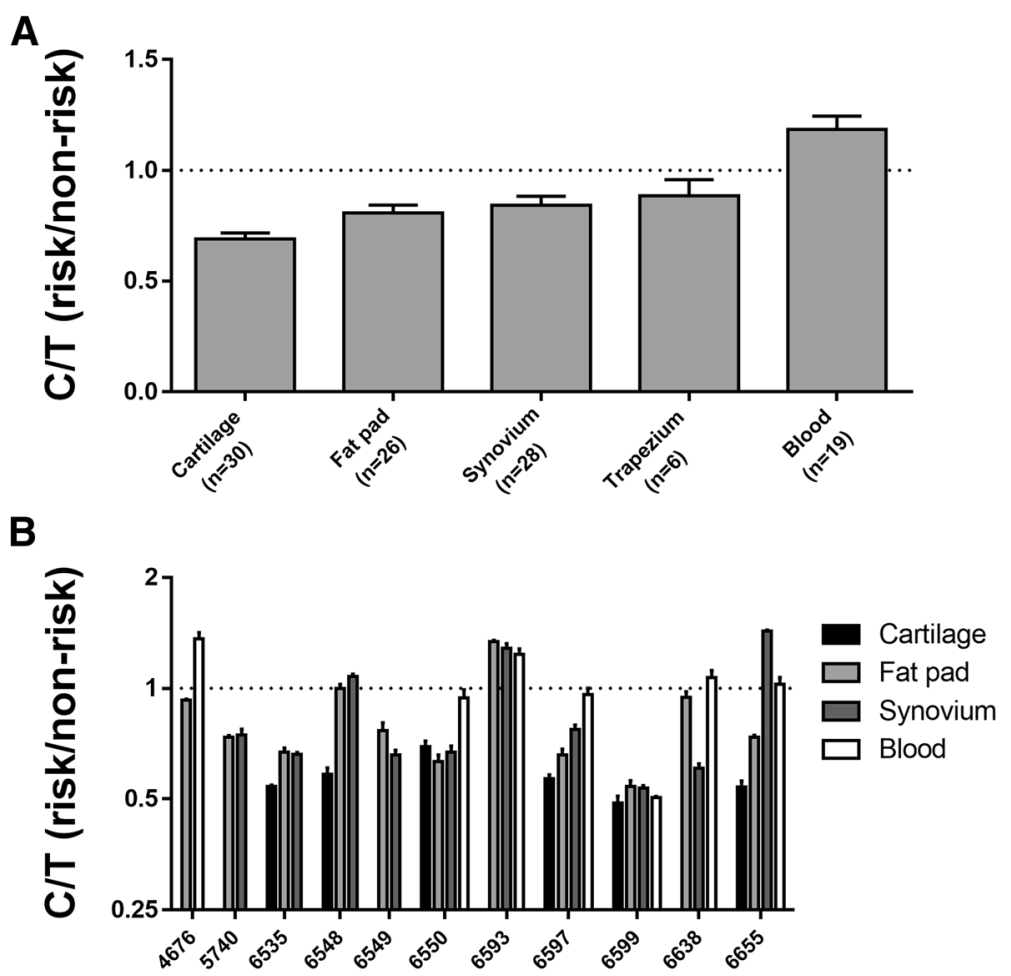

Fig. 3 Difference in AEl between tissues and within the tissues of the same patient. a The mean AEI value ( \pm SEM) was plotted for each of the five tissue types. $\mathbf{b}$ AEl analysis of 11 patients for whom samples were available from at least two tissues. Values are the mean \pm standard deviation (SD) AEl plotted for each individual in each tissue tested. Patients are designated by their anonymised ID number. In both plots, the broken horizontal line indicates a $C / T$ ratio of 1 , which is indicative of no allelic imbalance

transcription factor SOX-9; fold change $=2.83, P<0.01$ ), COL10A1 (encodes the $\alpha 1$ polypeptide chain of type $\mathrm{X}$ collagen; fold change $=2.17, P=0.05$ ), VEGFA (encodes vascular endothelial growth factor $\mathrm{A}$; fold change $=2.15$, $P<0.01$ ), MMP13 (encodes collagenase 3; fold change $=$ 1.18, $P<0.05$ ), ADAMTS4 (encodes the aggrecanase A disintegrin and metalloproteinase with thrombospondin motifs 5; fold change $=2.17, P<0.05$ ) and $B C L 2$ (encodes apoptosis regulator $\mathrm{Bcl}-2$; fold change $=1.51, P<0.05$ ). Therefore, in this model system, depletion of MGP transcript and of its protein in OA knee cartilage had a significant impact on genes encoding regulators and markers of cartilage homeostasis.

\section{Discussion}

Our initial experiments assessed the expression of MGP in multiple cell types from OA patients, with a focus on joint tissues. There was expression in all five of the joint tissue types tested, with the highest level being in cartilage. In a comparison between $\mathrm{OA}$ and non-OA cartilage (NOF samples), there was a greater expression in OA but a still abundant expression in non-OA cartilage (mean TPM values $>10,000$ and $>5000$, respectively). This implies that the expression of the gene is not caused by OA but that its level increases in response to OA, perhaps as an attempt to halt the disease process. In our MSC chondrogenesis models, we observed increased expression of MGP during cartilage formation, reiterating that expression of the gene is part of normal cartilage formation.

We next set out to replicate the $M G P$ allelic expression imbalance that the earlier study had reported on [8]. Using MGP transcript SNP rs4236, we also showed that the OArisk conferring $\mathrm{T}$ allele of rs4764133 correlated with reduced expression of the gene in patient cartilage. The degree of AEI was highly comparable between our report and the earlier study: $40.8 \%$ and $39.6 \%$ of the risk allele, respectively. In that earlier report, the authors had also observed reduced expression of the risk allele in subchondral bone. We did not investigate this tissue in our patients, but we did observe significant MGP AEI in fat pad and synovium tissue and in the same direction as observed in cartilage. However, the reduction in expression of the risk allele was greater in cartilage; $40.8 \%$ of the risk allele versus $44.8 \%$ in fat pad and $45.7 \%$ in synovium. Overall, this implies that the functional consequence of the OA risk mapping to this locus, namely reduced MGP expression, is impacting on multiple knee and hip joint tissues and that of those tissues tested; its effect is more profound in cartilage. We also investigated MGP expression and AEI of MGP in blood from OA patients. Matrix 

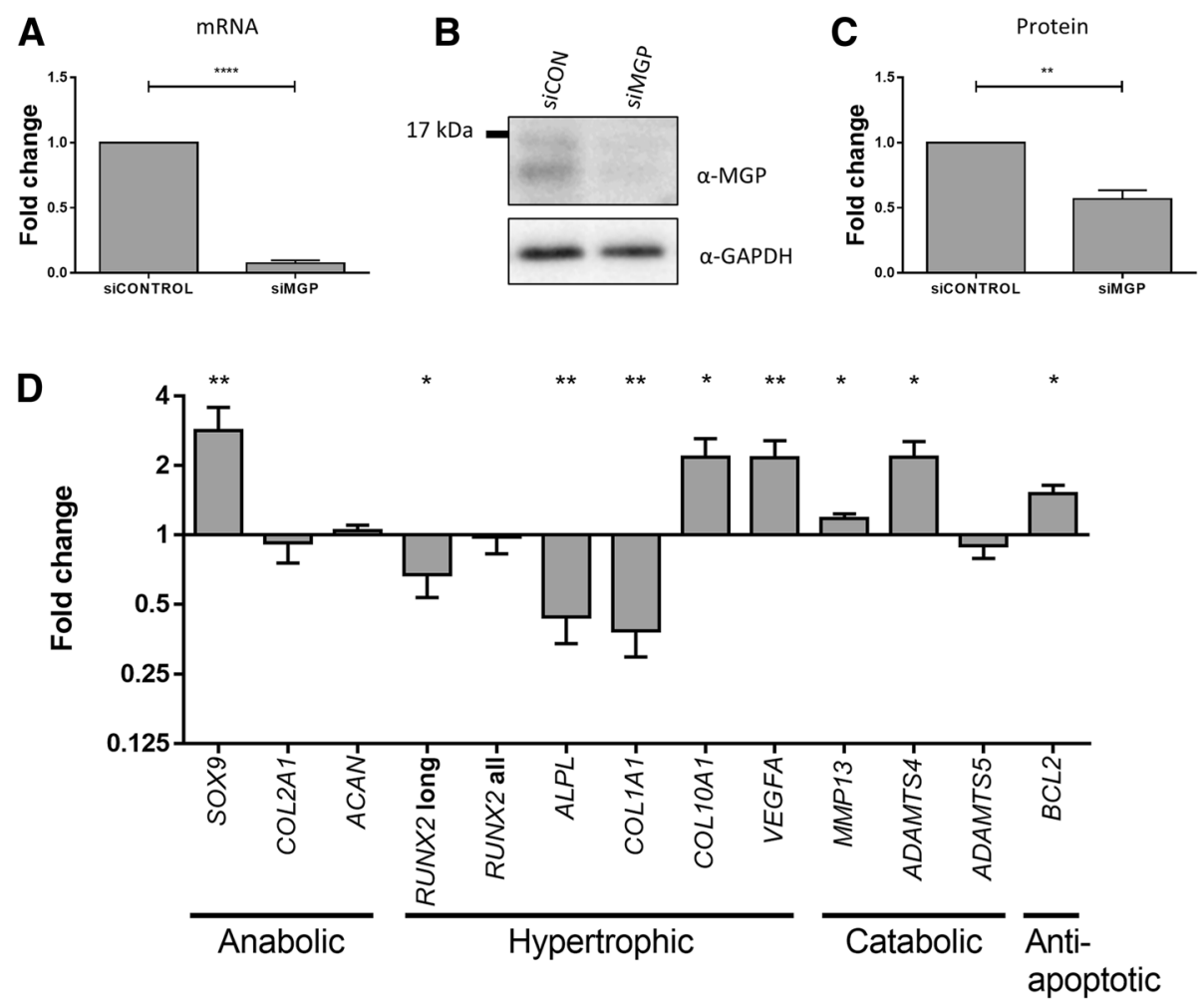

Fig. 4 Effects of knockdown of MGP in primary chondrocytes from patients with OA. a MGP knockdown with siRNA targeting MGP (siMGP) was carried out in cultured knee chondrocytes from nine OA patients, in comparison to the effects of a nontargeting siRNA control (siCONTROL). MGP mRNA levels were measured by qPCR. Values represent the mean \pm standard deviation (SD) fold change in expression compared to siRNA control, with the data combined for the patients. $\mathbf{b}$ Representative results from immunoblotting demonstrating depletion of matrix Gla protein. GAPDH was used as the loading control. c Fold change in matrix Gla protein level with the immunoblotting data combined for the patients. Values represent the mean \pm SD fold change compared to siRNA control. d Fold change in expression of genes following MGP knockdown, relative to that with the siRNA control. Values represent the mean \pm SD fold change in expression compared to siRNA control, with the data combined for the patients. ${ }^{*} P \leq 0.05 ;{ }^{*} P \leq 0.01 ;{ }^{* * *} P \leq 0.0001$

Gla protein is synthesised by vascular smooth muscle cells, where it inhibits vessel calcification [19], and by leucocytes, where it may have a role in modulating immune responses [20]. Expression of $M G P$ was readily detectable in our blood samples, and there was AEI but in the opposite direction to that seen in joint tissues, with an increased expression of the OA risk allele. Our interpretation of this is that the polymorphic DNA regulatory element that is mediating MGP AEI is utilised by joint tissue and blood cells but that it is acted on by different trans-acting factors between the cell types, leading to opposite effects on MGP expression. We have previously shown that the direction and degree of AEI at an OA risk gene can vary between different cell types that are concurrently collected from an OA patient [21]. Our MGP results support this earlier observation and emphasise the probable complexity behind the regulation of the expression of this gene. A search of the GWAS Catalog (https://www.ebi.ac.uk/gwas/) did not identify any associations between MGP polymorphisms and ectopic calcification diseases of the vasculature. Nevertheless, the functional effect that we have observed in blood may prioritise rs4764133 and its correlating SNPs for future analyses in such diseases.

In the GWAS that reported on MGP, rs4764133 was associated with hand $\mathrm{OA}$ and, as we have done, the investigators who reported that result then used joint tissues from patients who had undergone knee and hip arthroplasty to investigate MGP expression [8]. This is a pragmatic decision in that these surgical procedures are common, and as such, knee and hip joint tissues are readily available for research. In our study, in addition to knee and hip samples, we also managed to collect osteochondral tissue from patients who had undergone a trapeziectomy. This involves the removal of the trapezium joint that is located at the base of the thumb and within the wrist. From a skeletal perspective, trapezium more closely matches the phenotype used in the GWAS than knees or hips, in that it is a hand joint. Furthermore, the trapezium was one of the joints that was included in the measure of hand $\mathrm{OA}$ in the genetic study. MGP was expressed in our trapezium samples, but there was no significant AEI when the six trapezium patients who we 
were able to study were combined. There was however a trend and in the same direction as seen for the other joint tissues, namely reduced expression of the OA riskconferring allele.

By knocking down the gene to deplete the levels of matrix Gla protein, we finally modelled in cartilage chondrocytes the reduced expression of the MGP risk allele. This had a significant effect on a range of genes involved in cartilage homeostasis. We observed an increased expression of the master chondrogenic transcription factor gene SOX9, but this was not accompanied by an increased expression of two of its principal target genes, COL2A1 and ACAN, which code for the key structural components of cartilage, type II collagen and aggrecan, respectively. Instead, we observed an increased expression of the genes MMP13 and ADAMTS4, which code for enzymes that degrade these two proteins [22, 23]. We also observed an increased expression of COL1OA1 and VEGFA, which are markers of cartilage ossification $[24,25]$. These data suggest that depletion of matrix Gla protein may have a detrimental impact on chondrocytes, whereby several markers of catabolism, hypertrophy and ossification are elevated. We hypothesise that the observed increase in SOX9 expression may be a response to counter this by boosting anabolism. There are, however, some results from the knockdown experiment that do not align with this interpretation. For example, decreased expression of $A L P L$, which is also a marker of cartilage ossification [26]. Overall however, recapitulating the AEI by reducing the expression of MGP had clear effects on chondrocyte marker genes and demonstrates that, at least in this model system, alteration in the level of matrix Gla protein has functional consequences.

In addition to our functional studies, mutations in MGP have been described which cause calcification of the vascular system and cartilage. Keutel syndrome arises from MGP mutations and manifests as vascular calcification with skeletal abnormalities, and an MGP-deficient mouse model displays a similar phenotype [27]. Vitamin K, and MGP functionality, was implicated in OA when plasma vitamin $\mathrm{K}$ concentrations were correlated with radiographic signs of OA or pathological changes to the joint [28, 29]. Furthermore, MGP polymorphisms were significantly associated with OA in a candidate gene study investigating vitamin $\mathrm{K}$ supplementation in deficient individuals [30]. Taken together, there is now a growing body of evidence to support further exploration of MGP and its functional role in cartilage and matrix biology, and in the ectopic mineralisation process.

\section{Conclusions}

We have replicated and expanded on the study that correlated expression of $M G P$ with the OA association signal marked by SNP rs4764133. We confirm that the OA risk-conferring allele of this SNP demonstrates reduced expression in cartilage and in other joint tissues. We also show that in peripheral blood, this effect is reversed, highlighting complexity in the regulation of expression of the gene. In our RNA-seq data, $M G P$ was more highly expressed in OA versus non-OA cartilage. Combining this observation with our AEI observations, we conclude that in response to $\mathrm{OA}$, chondrocytes need to express more MGP but that in an individual who has inherited low expressing alleles of MGP, their capacity to boost the gene's expression is attenuated. As such, the disease is more likely to progress and less likely to resolve in these individuals. Our recapitulation by RNAi of the functional effect of this genetic signal had clear impacts on chondrocyte gene expression. As such, MGP knockdown is an appropriate model that could now be exploited to assess the capacity of interventions to overcome the genetic susceptibility at this locus.

\section{Additional files}

Additional file 1 Table S1. Details of the 165 osteoarthritis patients included in this study. (XLSX $15 \mathrm{~kb}$ )

Additional file $\mathbf{2}$ Table S2. Primers used for genotyping and AEI analysis of rs4236. (DOCX 16 kb)

\section{Abbreviations}

AEl: Allelic expression imbalance; GWAS: Genome-wide association scan; HACs: Human articular chondrocytes; LD: Linkage disequilibrium; MSC: Mesenchymal stem cells; NOF: Neck-of-femur fracture;

OA: Osteoarthritis; qPCR: Quantitative polymerase chain reaction; RNAi: RNA interference; siRNA: Short interfering RNA; SNP: Single nucleotide polymorphism; TPM: Transcripts per million

\section{Acknowledgements}

We thank Matt Barter and Maria Tselepi for providing us with access to cDNA from MSC chondrogenesis. We thank Andrew Skelton for assistance with the analysis of the RNA-seq data.

\section{Authors' contributions}

All authors were involved in drafting the article or revising it critically for important intellectual content, and all authors approved the final version to be published. CS and JL had full access to all of the data in the study and take responsibility for the integrity of the data and the accuracy of the data analysis. CS, LNR and JL were responsible for the study conception and design. CS and AER were responsible for the acquisition of data. CS, AER, $L N R$ and $J$ were responsible for the analysis and interpretation of data.

\section{Funding}

This work was supported by Versus Arthritis (grant 20771), by the Medical Research Council and Versus Arthritis as part of the Centre for Integrated Research into Musculoskeletal Ageing (CIMA, grant references JXR 10641, MR/P020941/1 and MR/R502182/1) and by the European Union's Seventh Framework Program for research, technological development and demonstration under grant agreement number No. 305815 (D-BOARD). Funds to purchase the pyrosequencer were provided by The Ruth and Lionel Jacobson Charitable Trust.

Availability of data and materials

RNA-seq data have been deposited in the NCBI Gene Expression Omnibus [accession no. GSE111358].

Ethics approval and consent to participate

Each donor provided verbal and written informed consent (REC reference number 14/NE/1212). Ethical approval was granted by the Newcastle and 
North Tyneside Research Ethics Committee (REC reference number 14/NE/ 1212).

\section{Consent for publication}

Not applicable.

\section{Competing interests}

The authors declare that they have no competing interests.

Received: 6 February 2019 Accepted: 6 June 2019

Published online: 18 June 2019

\section{References}

1. Loeser RF, Goldring SR, Scanzello CR, Goldring MB. Osteoarthritis: a disease of the joint as an organ. Arthritis Rheum. 2012;64:1697-707.

2. Kendzerska T, Jüni P, King LK, Croxford R, Stanaitis I, Hawker GA. The longitudinal relationship between hand, hip and knee osteoarthritis and cardiovascular events: a population-based cohort study. Osteoarthr Cartil. 2017;25:1771-80.

3. Reynard LN. Analysis of genetics and DNA methylation in osteoarthritis: what have we learnt about the disease? Semin Cell Dev Biol. 2017;62:57-66.

4. Styrkarsdottir U, Helgason H, Sigurdsson A, Norddahl GL, Agustsdottir AB, Reynard LN, Villalvilla A, Halldorsson GH, Jonasdottir A, Magnusdottir A, et al. Whole-genome sequencing identifies rare genotypes in COMP and CHADL associated with high risk of hip osteoarthritis. Nat Genet. 2017;49:801-5.

5. Zengini E, Hatzikotoulas K, Tachmazidou I, Steinberg J, Hartwig FP, Southam L, Hackinger S, Boer CG, Styrkarsdottir U, Gilly A, et al. Genome-wide analyses using UK Biobank data provide insights into the genetic architecture of osteoarthritis. Nat Genet. 2018;50:549-58.

6. Styrkarsdottir U, Lund SH, Thorleifsson G, Zink F, Stefansson OA, Sigurdsson JK, Juliusson K, Bjarnadottir K, Sigurbjornsdottir S, Jonsson S, et al. Metaanalysis of Icelandic and UK data sets identifies missense variants in SMO, IL11, COL11A1 and 13 more new loci associated with osteoarthritis. Nat Genet. 2018:50:1681-7.

7. Tachmazidou I, Hatzikotoulas K, Southam L, Esparza-Gordillo J, Haberland V, Zheng J, Johnson T, Koprulu M, Zengini E, Steinberg J, et al. Identification of new therapeutic targets for osteoarthritis through genome-wide analyses of UK Biobank data. Nat Genet. 2019; in press.

8. den Hollander W, Boer CG, Hart DJ, Yau MS, Ramos YF, Metrustry S, Broer L, Deelen J, Cupples LA, Rivadeneira F, et al. Genome-wide association and functional studies identify a role for matrix Gla protein in osteoarthritis of the hand. Ann Rheum Dis. 2017;76:2046-53.

9. Gheorghe SR, Crăciun AM. Matrix Gla protein in tumoral pathology. Clujul Med. 2016;89:319-21.

10. Thomas S, Browne H, Mobasheri A, Rayman MP. What is the evidence for a role for diet and nutrition in osteoarthritis? Rheumatology (Oxford). 2018; 57(suppl 4):iv61-74.

11. Gee F, Rushton MD, Loughlin J, Reynard LN. Correlation of the osteoarthritis susceptibility variants that map to chromosome 20q13 with an expression quantitative trait locus operating on $\mathrm{NCOA} 3$ and with functional variation at the polymorphism rs1 16855380. Arthritis Rheumatol. 2015;67:2923-32.

12. Rice SJ, Aubourg G, Sorial AK, Almarza D, Tselepi M, Deehan DJ, Reynard LN, Loughlin J. Identification of a novel, methylation-dependent, RUNX2 regulatory region associated with osteoarthritis risk. Hum Mol Genet. 2018; 27:3464-74.

13. Farhang N, Brunger JM, Stover JD, Thakore PI, Lawrence B, Guilak F, Gersbach CA, Setton LA, Bowles RD. CRISPR-based epigenome editing of cytokine receptors for the promotion of cell survival and tissue deposition in inflammatory environments. Tissue Eng Part A. 2017;23:738-49.

14. Barter MJ, Gomez R, Hyatt S, Cheung K, Skelton AJ, Xu Y, Clark IM, Young DA. The long non-coding RNA ROCR contributes to SOX9 expression and chondrogenic differentiation of human mesenchymal stem cells. Development. 2017;144:4510-21.

15. Shepherd C, Skelton AJ, Rushton MD, Reynard LN, Loughlin J. Expression analysis of the osteoarthritis genetic susceptibility locus mapping to an intron of the MCF2L gene and marked by the polymorphism rs11842874. BMC Med Genet. 2015;16:108.

16. Love Ml, Huber W, Anders S. Moderated estimation of fold change and dispersion for RNA-seq data with DESeq2. Genome Biol. 2014;15:550.

17. Bui C, Barter MJ, Scott J-L, Xu Y, Galler M, Reynard LN, Rowan AD, Young D. CAMP response element-binding (CREB) recruitment following a specific $\mathrm{CpG}$ demethylation leads to the elevated expression of the matrix metalloproteinase 13 in human articular chondrocytes and osteoarthritis. FASEB J. 2012;26:3000-11.

18. Schneider CA, Rasband WS, Eliceiri KW. NIH Image to ImageJ: 25 years of image analysis. Nat Methods. 2012:9:671-5.

19. Bjorklund G, Svanberg E, Dadar M, Card DJ, Chirumbolo S, Harrington DJ, Aaseth J. The role of matrix Gla protein (MGP) in vascular calcification. Curr Med Chem. in press.

20. Viegas CS, Costa RM, Santos L, Videira PA, Silva Z, Araújo N, Macedo AL, Matos AP, Vermeer C, Simes DC. Gla-rich protein function as an anti-inflammatory agent in monocytes/macrophages: implications for calcification-related chronic inflammatory diseases. PLoS One. 2017;12:e0177829.

21. Wilkins JM, Southam L, Price AJ, Mustafa Z, Carr A, Loughlin J. Extreme context specificity in differential allelic expression. Hum Mol Genet. 2007;16:537-46.

22. Li H, Wang D, Yuan Y, Min J. New insights on the MMP-13 regulatory network in the pathogenesis of early osteoarthritis. Arthritis Res Ther. 2017;19:248.

23. Kelwick R, Desanlis I, Wheeler GN, Edwards DR. The ADAMTS (a Disintegrin and Metalloproteinase with Thrombospondin motifs) family. Genome Biol. 2015;16:113.

24. Tsang KY, Chan D, Cheah KS. Fate of growth plate hypertrophic chondrocytes: death or lineage extension? Develop Growth Differ. 2015;57:179-92

25. Patil AS, Sable RB, Kothari RM. Occurrence, biochemical profile of vascular enothelial growth factor (VEGF) isoforms and their functions in endochondral ossification. J Cell Physiol. 2012;227:1298-308.

26. Millán JL. The role of phosphatases in the initiation of skeletal mineralization. Calcif Tissue Int. 2013;93:299-306.

27. Luo G, Ducy P, McKee MD, Pinero GJ, Loyer E, Behringer RR, Karsenty G. Spontaneous calcification of arteries and cartilage in mice lacking matrix GLA protein. Nature. 1997;6:78-81.

28. Neogi T, Booth SL, Zhang YQ, Jacques PF, Terkeltaub R, Aliabadi P, Felson DT. Low vitamin $\mathrm{K}$ status is associated with osteoarthritis in the hand and knee. Arthritis Rheumatol. 2006;54:1255-61.

29. Shea MK, Kritchevsky SB, Hsu FC, Nevitt M, Booth SL, Kwoh CK, McAlindon $T E$, Vermeer $C$, Drummen N, Harris TB, et al. The association between vitamin $\mathrm{K}$ status and knee osteoarthritis features in older adults: the Health, Aging and Body Composition Study. Osteoarthr Cartil. 2015;23:370-8.

30. Misra D, Booth SL, Crosier MD, Ordovas JM, Felson DT, Neogi T. Matrix Gla protein polymorphism, but not concentrations, is associated with radiographic hand osteoarthritis. J Rheumatol. 2011;38:1960-5.

\section{Publisher's Note}

Springer Nature remains neutral with regard to jurisdictional claims in published maps and institutional affiliations.
Ready to submit your research? Choose BMC and benefit from:
- fast, convenient online submission
- thorough peer review by experienced researchers in your field
- rapid publication on acceptance
- support for research data, including large and complex data types
- gold Open Access which fosters wider collaboration and increased citations
- maximum visibility for your research: over $100 \mathrm{M}$ website views per year
At BMC, research is always in progress.
Learn more biomedcentral.com/submissions 